\title{
Zur Funktion von Bericht und Reportage in der Kirchenzeitung
}

\author{
von K. Rüdiger Durth
}

\section{Eine überbolte Mabnung}

Kaspar Stieler, auch als "Spate" bekannt, wurde von einer bitteren Erfahrung geplagt. In seiner 1695 veröffentlichten ersten großen Zeitungslehre - sie trägt den schönen, angesichts heutiger Medienkritik kaum mehr denkbaren Titel „Zeitungs Lust und Nutz" - forderte er die Geistlichen auf, ihre sonntäglichen Zuhörer zu ermahnen, die Zeitungen nicht mit in die Kirche zu nehmen und darin zu lesen: „Am schändlichsten aber stehet, wenn sie es selber tuhn oder des Orts Obrigkeit unter der Orgel und denen Gesängen das Avisenblatt herausnimmt und auf der Porkirche öffentlich durchschauet."

Die Mahnung ist überholt. Wohl kaum jemand kommt heute auf die Idee, während des Gottesdienstes "Bild am Sonntag" zu lesen, hingegen würden sich die Verlage und Redaktionen der Kirchenzeitungen freuen, wenn die Geistlichen beispielsweise während der Woche öfters in die „Zeitung von Katholiken für Katholiken" schauten, wenn sich die Gläubigen an die „Aufforderung" der Pastoralinstruktion "Communio et Progressio“ hielten, "die katholische Presse, die diesen Namen wirklich verdient, regelmäßig zu verfolgen" (Abschnitt 140).

Ober die Ursachen von Stagnation oder gar Rückgang der - katholischen wie evangelischen - Kirchenzeitungen ist in den zurückliegenden Jahren viel nachgedacht worden. Reformen und inhaltliche Verbesserungen sind nicht zu bestreiten, aber der Auftrag von "Communio et Progressio“ an die katholische Presse, auch "Spiegel zu sein, der die Welt reflektiert, und Licht, das ihr den Weg zeigt" (Abschnitt 138), ist weithin noch ein fernes Ziel geblieben. Kein Zweifel: viele Kirchenzeitungen sind langweilig, weil sie zu sehr "offizielles Organ“ des Bistums und zu wenig eine Zeitung der Kirche als Gemeinschaft der Gläubigen sind.

Was "Communio et Progressio" dem Redakteur mit viel Verständnis zubilligt, nämlich "die Nachrichten vielfach einem gehetzten und unkonzentrierten Publikum so lebendig vermitteln (zu müssen), daß es angesprochen und gefesselt wird" (Abschnitt 40), wird dem Journalisten der Kirchenzeitung nicht zugestanden. Schließlich ist idas Bistumsblatt nicht „Bild“. Schon richtig - nur fragt sich, ob nicht sein Leser auch "gehetzt und unkonzentriert" ist, müde von den Strapazen des Alltags, "verwöhnt" von "Bild", abonnierter Lokalzeitung und Fernsehen? Sieht man ab von den Alten und Kranken, die Zeit haben und das gesamte Bistumsblatt lesen - eingedenk der „Spaten"-Mahnung:

„Es ist etliche mal gesagt worden, daß man die Zeitung völlig auslesen und nicht überhüpfen solle. Es sind ihrer viel, welche, wann sie eine Zeitung in die Hände kriegen, nur nachsehen, ob etwas von Wien oder aus diesem und jenem Feldlager darinnen enthalten sey? Das laufen sie geschwinde durch und werfen hernach die Zeitung weg, fluchen auch noch wol drüber, daß nicht eine Schlacht vorgangen und

K. Rüdiger Durth ist Pastor in der Evangelischen Kirche des Rheinlandes und hauptberuflich Redakteur der „Bonner Rundschau“. 
der Feind überwunden ist. Solche Leute verstehen nicht, was Zeitungen seyn und auf sich haben; sondern verraten bloß ihren Fürwitz und scheltwürdige Neugierigkeit ...

Es muß aber auch eine Achthabung bey der Zeitungs-Lesung seyn. Großes mit Kleinem zu vergleichen; Gehestu gleich in die Kirche und hörest den Pfarrer vom Anfange bis zum Ende predigen; Wann du nicht recht aufmerkest und eigendlich Achtung giebest, was geprediget wird; so hättestu wol zu Hause bleiben mögen. Ist doch sonst in dem menschlichen Leben alles verloren, wenn man nicht bedenket, was man tuht; Derowegen, wenn es dir ein Ernst ist, aus Zeitungen klug zu werden; so haben deine Gedanken beysammen: denn du hast es nötig, zumal üm der mancherley Sachen willen, so aus Osten und Westen berichtet werden."

\section{Sensationen finden nicht statt}

Im Gegensatz zur Tages- oder politischen Wochenzeitung hat die Kirchenzeitung den Nachteil, daß sie so gut wie nie mit Nachrichten-Sensationen aufwarten kann: Die Abdankung eines Bischofs, die Erhebung eines Geistlichen zum Kardinal, der Streit um "Adveniat", interessante kirchliche "Geheimpapiere" — das alles lesen wir zuerst in der Zeitung. Und selbst ein "Theologenkrach", etwa um oder mit Hans Küng, Horst Hermann, nur um zwei Namen zu nennen, findet in den säkularen Medien statt - weil die Kirchenzeitung differenzierter argumentieren muß, weil sie auch die Meinungen zu Wort kommen lassen muß, die einen „Skandal“ — was das auch immer sein mag - relativieren, ihm den Wind aus den Segeln nehmen.

Nur selten kommen Kirchenzeitungen mit ihren Kommentaren - oft besser und fundierter als Kommentare in überregionalen Blättern - in die "Presseschau" von Tageszeitung, Hörfunk oder Fernsehen. Das hat u.a. die Folge, daß die Kirchenzeitung in der Offentlichkeit weithin unbekannt bleibt.

Unter den Journalisten selbst nimmt der Redakteur einer Kirchenzeitung oft eine Sonderstellung ein - zwischen Journalist und Werkszeitung, Lobby und Propagandist der angeblich viel $\mathrm{zu}$ einflußreichen Kirche. Vorurteile, die auch im Journalismus recht zählebig sind.

Dem Journalisten der Kirche fehlt die Offentlichkeit im weiten Sinn, die Anerkennung unter den Berufskollegen in der Kirche, und im Blick auf die Kirche, über und für die er schreibt, hat er mit den gleichen Schwierigkeiten zu kämpfen.

\section{Journalistische Stilformen nicht nur Selbstzweck}

Die Kirchenzeitung muß nicht langweilig sein und kann sehr wohl ohne Sensationen auskommen - vielleicht ist das sogar ihre Chance in einer Zeit, in der sich Zeitungen und Zeitschriften gegenseitig mit Sensationen zu übertreffen versuchen und das Fernsehen das Jahr 1978 unter das Motto „noch mehr Unterhaltung“ gestellt hat. Die Chance besteht darin, den Menschen hineinzunehmen in einen Bereich der ganz wesentlich zum Menschen gehört: Glauben, Meditation, Gemeinschaft, Sinnfrage, Mut zum Handeln für den nahen und fernen Nächsten. Hineinzunehmen also in die Kirche, die ja stets mehr ist als Bischof oder Institution.

Das heißt, die Kirchenzeitung hat die Aufgabe, über die Vielfalt der Kirche mit der Vielfalt journalistischer Stilformen $\mathrm{zu}$ informieren. In seinem Grußwort zum 25jährigen Bestehen der Katholischen Nachrichtenagentur (KNA) am 14. November 
1977 hat der Vorsitzende der Deutschen Bischofskonferenz, Joseph Kardinal Höffner, das Problem so umrissen:

„Der Auftrag der Kirche heißt Verkündigung. Zwar sieht die Kirche in den Medien - eingeschlossen die Kirchenpresse - keine ,zweite Kanzel‘. Aber von ihrem Auftrag her muß sie auch das über die Medien mit der Gesellschaft geführte Gespräch als einen Teil ihrer Verkündigung sehen. Allerdings - und hier ist der Lernprozeß noch nicht abgeschlossen - ist nicht alles, was innerhalb der Kirche geschieht, auf den Verkündigungsauftrag unmittelbar zurückzuführen. An dieser Stelle wird das Gespräch zwischen Kirche und Medien noch intensiv fortgesetzt werden müssen."

Doch ein Blick in die Kirchenpresse zeigt, daß die Möglichkeiten der journalistischen Stilformen nur unzureichend genutzt werden. Statement und Dokumentation z. B. bischöflicher Worte, Reden, Predigten, Grundsatzerklärungen - stehen ebenso im Vordergrund wie beschauliche oder fromme Geschichten, deren Mittelpunkt lauter gute Menschen bilden. Einschließlich der kämpferischen Artikel - meist gegen etwas in der Gesellschaft - und der offiziellen Fotos wird dem Leser der Kirchenzeitung eine heile Kirche in einer heillosen Welt vermittelt. Und das oft genug in einer Sprache, die man gemeinhin Kirchensprache nennt und die sich nur schlecht mit der Zeitungssprache verträgt. Letztere übrigens ist besser, als ihre lautstarken Kritiker, allen voran Friedrich Nietzsche, behaupten: „Schweinedeutsch - Verzeihung. Zeitungsdeutsch."

Immerhin ist das Sprachproblem in der Kirche selbst bekannt. Joseph Kardinal Höffner in seinem bereits oben erwähnten Grußwort zum KNA-Jubiläum: „Ein spezielles Problem der Kirche in ihrem Verhältnis zu den Medien ist die Sprache. Die Sprache der Kirche, so heißt es, müsse erst übersetzt wenden, um auch die Sprache der Medien zu sein, und gerade diese ,Ubersetzung' sei eine Quelle für Fehldeutungen und Mißverständnisse. Dieses Problem darf nicht verkannt werden, auch wenn hier

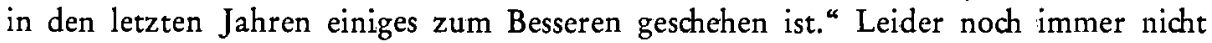
genug.

Man vermißt in der Kirchenzeitung die gute Mischung der journalistischen Stilformen wie Nachricht, Bericht, Hintergrundbericht, Reportage, Bildbericht, Interview (als tatsachenorientierte Formen) und Kommentar, Rezension, Glosse, geistliche Betrachtung, religiöse Kurzgeschichte, Gedicht, christliche Erzählung (als meinungsorientierte Formen).

Die journalistischen Stilformen sind nämlich nicht nur eine Formsache, sondern bedingen auch einen entsprechenden Inhalt. Die Frage, die sich immer wieder stellt, ist: Welche Form wähle ich für welches Thema? Von der richtigen Beantwortung und anschließend der richtigen Handhabung - hängt nicht unwesentlich die Attraktivität der Kirchenzeitung und ihres Inhaltes ab.

Viele Kirchenzeitungen setzen ihren Ehrgeiz in die Gewinnung prominenter kirchlicher Autoren, deren Manuskript anschließend selbstverständlich nicht geändert werden darf. So notwendig und richtig es ist, solche Autoren in seinem Blatt vertreten zu wissen, so ist das Risiko doch erheblich, nämlich, daß der Leser letztlich nicht viel davon hat. Und der Artikel wird nicht dadurch besser, daß man im Vorspann auf die Bekanntheit des Mannes (Frauen sind auch hier nur sehr selten vertreten) hinweist - er wird dem Leser nicht dadurch bekannter, daß die Redaktion ihn bekannt nennt. Vergessen wir nicht: die meisten Leser einer Kirchenzeitung wissen nicht, wer Hans Küng, Karl Rahner, Johann Baptist Metz sind. Welche 
Möglichkeiten aus der Vielfalt der journalistischen Stilformen der Bericht und die Reportage für die Kirchenzeitung bieten, das wollen wir im folgenden untersuchen. Gleichzeitig lassen wir einige praktische Tips einfließen.

\section{Vom Jargon und anderen Argernissen}

$\mathrm{Da}$ sowohl der Bericht als auch die Reportage wesentlich von dem Stil ihres Autors abhängen, wollen wir uns zunächst ein wenig mit dem Problem der Sprache beschäftigen. Ein Problem, das übrigens für die übrigen journalistischen Stilformen ebenfalls gilt.

Zeitungsdeutsch oder Kirchensprache in der Kirchenzeitung? Die Antwort muß eindeutig lauten: Zeitungsdeutsch. Daß die Sprache der Kirche auch im Volk fester verankert ist als viele meinen, zeigte 1977 die heftige Kontroverse in der evangelischen Kirche um den neuen revidierten Text der Lutherbibel. Die auf Zeitgeist bedachten Übersetzer wollten plötzlich in der Bergpredigt das Licht nicht mehr unter den Scheffel, sondern unter den Eimer stellen. Nach einem orkanartigen Protest war der Eimer buchstäblich im Eimer und es bleibt beim Scheffel. Luthers Wort „Die Sprachen sind die Scheiden, darinnen die Schwerter des Geistes verborgen ruhen", bewahrheitete sich auf seine Weise.

Aber dies ist kein Argument für die Kirchensprache in der Kirchenzeitung. Bibel und Zeitung sind zweierlei Dinge, und die Kirchenzeitung unterliegt der Sach-, nicht der Eigengesetzlichkeit des Journalismus. Daß im Problem der Sachgesetzlichkeit ethischer Sprengstoff verborgen ist, soll hier nur erwähnt sein.

Die Kanzelsprache hat in den Spalten der Kirchenzeitung nichts zu suchen. Sie wirkt geschrieben fremd, langatmig, pathetisch, gekünstelt - und auf den der Kanzel entfremdeten Leser leer, nichtssagend. Schon um seinetwillen muß auf diese Sprache verzichtet werden, weil die Kirchenzeitung ihren wohl verstandenen missionarischen Auftrag nicht aufgeben darf.

Der Jargon hat ebenfalls in der Zeitung nichts zu suchen, obwohl nicht wenige davon überzeugt sind, er sei modern und komme gut an, vor allem bei den jüngeren Lesern. Uber Gott und die Kirche kann man weder im Jargon reden noch schreiben - Gott erniedrigte sich zwar bis in den Tod, nicht aber in die Gassensprache - noch erreicht man damit den Menschen. Jargon verärgert den älteren Leser und läßt den jüngeren Anbiederung vermuten, die er ablehnt. Die Sprache muß überzeugen, nicht auf eine falsch verstandene Vertraulichkeit bauen.

Eng mit der Kanzelsprache hängt das Problem zusammen, daß man sich unbewußt schnell mit seinem Stil der Sprache anpaßt, die in dem Bereich gesprochen wird, über den man berichtet - also der Kirche. Höflichkeitsfloskeln, nichtssagende Attribute, Konjunktive, Wenn-und-Aber-Formulierungen, Schachtelsätze, alter Wortschatz schleichen sich ein.

Gerade für den kirchlichen Journalisten stellt sich das Ưbersteigerungsmoment ein, das Klischees zur Folge hat. Nichts aber tötet die Sprache mehr als Sprachklischees - die auch dadurch entstehen können, daß man sich nicht mehr um die Sprache im weiten Sinn kümmert, also anhand der Literatur, Dichtung etc. seinen eigenen Stil überprïft und fortentwickelt.

Ebenso ist vor einer Sprache der Abkürzungen zu warnen, die mit dem S. E. (kein Leser kann etwas damit anfangen) vor dem Bischofstitel anfängt (und ohnehin in der 
Zeitung nichts zu suchen hat) und beim PGR, dem Pfarrgemeinderat, aufhört. Sollte man sich schon bemühen, in der gesprochenen Sprache Abkürzungen zu vermeiden, so gilt dies ganz besonders für die geschriebene. Abkürzungen sind unbarmherzig, täuschen Herrschaftswissen vor, auf das der Leser sicherlich gern verzichtet.

Zeitungssprache ist eine „Berufssprache“ (Dovifat), die sich durch kurze und klare Sätze auszeichnet. Sie allein bringen Spannung in die journalistischen Stilformen und ermöglichen einen klaren Aufbau. Mit Dovifat sind Substantivierungen (der Nestor der deutschen Publizistik bezeichnete sie mit Recht als "Laster") ebenso zu vermeiden wie Zustandswörter. Der Konjunktiv läßt sich in den meisten Fällen vermeiden, gleiches gilt für die Hilfsverben, die offensichtlich auch im Journalismus unsterblich sind.

Je intensiver der kirchliche Journalist in der Kirche selbst lebt, desto eher gelingt es ihm, sie lebendig, überzeugend und einladend (warum nicht?) in den Spalten der Kirchenzeitung darzustellen. Es sei in diesem Zusammenhang an Goethe erinnert: "Wenn ihr's nicht fühlt, ihr werdet's nicht erjagen."

\section{Die Kirche ist reich an Berichten}

Der Bericht als der "umfangreichere Zwillingsbruder der Nachricht" (von La Roche) zählt auch in der Kirchenzeitung zu den wichtigsten Stilformen. Sein Aufbau entspricht dem der Meldung: Das Wichtigste an den Anfang und Beantwortung der fünf berühmten journalistischen W's: Wer, wann, wo, was, wie (warum)? Was in der Meldung in einem Satz ausgeführt wird, stellt im Bericht einen oder mehrere Absätze dar. Eingeleitet wird der Bericht durch eine knappe Zusammenfassung, auch Vorspann (im Amerikanischen lead) genannt.

Die Grenzen des Berichts zu Hintergrundbericht und Reportage sind fließend. In den Wochenzeitungen setzt sich immer mehr der Hintergrundbericht durch, der kommentierend ein Ereignis in seinen Gesamtzusammenhang stellt.

Wie die Nachricht (Meldung) muß auch der Bericht aktuell sein. Ausgehend von der lateinischen Grundbedeutung actualis = wirksam ergibt sich für die Kirchenzeitung folgende Aktualität: Neue Ereignisse in Kirche und Gesellschaft, die für den Leser der Kirchenzeitung von allgemeiner Bedeutung sind. Themen, die von der Redaktion

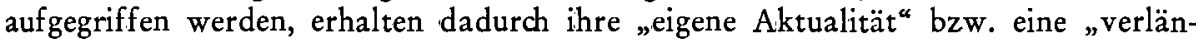
gerte Aktualität", wenn sie fortlaufend behandelt werden, etwa im Blick auf kirchliche Aktionen wie "Adveniat", "Misereor" oder die Auseinandersetzung mit wichtigen gesellschaftlichen Tendenzen, die in das Leben der Christen eingreifen.

$\mathrm{Da}$, wie gezeigt, die Kirchenzeitung ohne Sensationen auskommen muß, stellt sich für sie die Themenwahl als besonders schwierig dar. Grundsatz: Die Themen der Berichte müssen möglichst lesernah sein, d.h. dem Alltag der Kirche abgelauscht werden. Gerade der Alltag, der in den säkularen Massenkommunikationsmitteln so gut wie gar nicht vorkommt, ermöglicht dem Leser die Identifikation - d. h. er erkennt sich wieder in seinen Sorgen und Nöten, seinen Fragen, Hoffnungen, Enttäuschungen.

Zahlreiche offizielle Verlautbarungen der Amtskirche lassen sich dem Leser als zusammengefaßter Nachrichtenbericht besser verdeutlichen als durch den Abdruck des 
vollen oder auszugsweisen Wortlauts. Die Kirchenzeitung eignet sich nicht als „Bulletin“, und nur in den wenigsten Fällen ist aus der Sicht des Journalisten der Abdruck des Wortlauts als Dokumentation gerechtfertigt.

Ich vermisse in vielen Kirchenzeitungen Berichte über Konflikte und unterschiedliche Positionen innerhalb eines Bistums oder einer Region. Der Leser aber weiß selbst, daß auch die Kirche in der Welt keine heile Kirche ist. Der Bericht eignet sich als Stilform durchaus, den Forumscharakter einer Kirchenzeitung deutlich zu machen.

Das ist keine Aufforderung zum Konflikt-Journalismus. Im Gegenteil, wir sollten uns von dieser These nicht anstecken lassen. Aber Berichte über die vielfältigen, pluralistischen Strömungen innerhalb der Kirche tragen wesentlich zur inhaltlichen Verbesserung bei.

Die Kirche "spielt" sich wesentlich in persönlichen Beziehungen $a b-$ zur Ortsgemeinde, zur Messe, zur kirchlichen Gemeinschaft in allen ihren Formen. Berichte über den Alltag eines Priesters, einer Nonne, einer Schwester, eines Religionslehrers das alles hat noch Seltenheitscharakter. Gleiches gilt für die stillen und doch so wirkungsvollen Reformen, die sich ständig in einer lebendigen Kirche vollziehen. Hier kann der Bericht gute Dienste leisten und die notwendigen Informationen vermitteln. Die Kirche ist viele Berichte wert.

\section{Die Reportage - die Kirche "wieder hinbringen"}

Die Reportage, so belehrt uns der Große Duden, ist „ein um Objektivität bemühter Erlebnisbericht eines Reporters. Die Reportage soll den Leser das Ereignis miterleben lassen“. Das Wort stammt aus dem Französischen und bedeutet in seiner Verbform "wieder hintragen, wieder hinbringen". Im Blick auf unser Thema bedeutet also die journalistische Stilform Reportage, die Kirche wieder hinzubringen - zum Leser selbstverständlich.

Die Grenzen zwischen Bericht und Reportage sind fließend geworden, vor allem zum Erlebnis- und Hintergrundbericht. Die Reportage selbst verfügt über fließende Grenzen zum Report, Feabure, Porträt. Sie will durch die Kunst ihrer Darstellung den Leser in ein aktuelles, allgemein interessierendes Thema oder Ereignis führen, weshalb sich das Präsens als wichtigste Zeitform für die Darstellung eignet. Durch anschauliche Details (die zugleich die Authentizität fördern), Monologe und Dialoge, historische Rückblicke sowie Ausblicke in die Zukunft kann der Journalist anhand ausgewählter Ereignisse, Personen oder Sachthemen vom Allgemeinen zum Besonderen - besser noch vom Besonderen zum Allgemeinen - führen. Die einzelnen "Stücke“ werden so zu einem Ganzen "montiert ${ }^{\text {“ }}$ - zu einer Szene, einem Bild. Die Welt der Kirche erscheint dem Leser plötzlich wie in einem Brennglas. Er läßt sich hineinführen in Glaube und Kirche.

Das setzt Erlebnisfähigkeit des Journalisten voraus. Erleben aber läßt sich die Kirche nur dann, wenn man sich in ihr engagiert. Und von welchem Journalisten kann dies inehr vorausgesetzt werden als vom kirchlichen Redakteur? Dies ist nicht identisch mit der Aufgabe von Distanz, die auch vom kirchlichen Journalisten erwartet werden darf. Vielmehr wird sich der engagierte christliche Journalist von selbst um Distanz bemühen, die in seiner Aufgabe, nicht in seinem eigenen kirchlichen Betroffensein liegt. Erleben kann außerdem nur der etwas, der das Staunen nicht verlernt hat: das Staunen über das Leben in der Kirche mit ihrem Reichtum menschlicher Gaben 
und Phantasie für Gott; über das Handeln Gottes in dieser unserer Welt und seiner Kirche, die wir so gern trennen, obwohl sie doch zusammengehören.

Was für den Bericht in der Kirchenzeitung gilt, gilt auch für die Reportage: Die Themenwahl ist von besonderer Bedeutung. Themen für Berichte können auch Themen für Reportagen sein, wobei die Reportage den Vorteil hat, daß sie von der

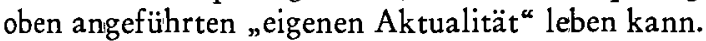

Im Volksbewußtsein sind die Evangelien des Neuen Testamentes viel tiefer verankert und bekannt als etwa die theologisch schwierigen Briefe des Apostels Paulus. Warum? Weil in den Evangelien viel erzählt wird, weil sie sich - die Formgeschichtler unter den Theologen bitte ich um Verzeihung - aus lauter Reportagen zusammensetzen. Und aus Berichten. Gekennzeichnet durch einfache und verständliche Sprache, Monologe und Dialoge, Gleichnisse, Szenenbeschreibungen. All das aber wird in den Redaktionszimmern vieler Kirchenzeitungen immer wieder übersehen, vergessen, erst gar nicht wahrgenommen. Die Bibel als journalistisches Lehrbuch? Auch das - für kirchliche Journalisten.

Was Martin Luther den Pfarrern empfohlen hat, sollten die Redakteure von Kirchenzeitungen nicht weniger beherzigen: "dem Volk aufs Maul zu schauen“. Das Ergebnis solchen "dem Volk aufs Maul Schauens" wird in den Redaktionen sicherlich keineswegs nur eitle Freude auslösen, aber mit Hilfe der Reportage zu wesentlichen Verbesserungen führen.

\section{Auch die Sünde ist eine Reportage wert}

Auch die Sünde ist eine Reportage wert. In der Kirchenzeitung. Denn Schuld und Sünde zählen zu den Grundbedingungen menschlichen Lebens, die im Alltag erfahren und immer schwieriger bewältigt werden. Von theologischen, philosophischen oder gesellschaftskritischen Betrachtungen abgesehen kommen sie jedoch in den Spalten der Kirchenzeitungen kaum vor. Der Leser aber findet sich dort kaum mit seinen Problemen wieder, denn die Sünde zeigt sich meist im einfachen Gewand. Wie kann sie aber überwunden werden - das ist ja die einzige theologisch legitime Lösung? Die populäre Lebensberatung reicht allein nicht aus.

Wichtig ist hingegen, daß die Kirchenzeitung Schuld und Sünde im konkreten Leben des Menschen nachgeht, sie ernst nimmt und dem Leser als selbsterfahrene Wirklichkeit vor Augen führt. Nur wenn man um die Konflikte weiß, lassen sie sich auch bewältigen.

Mangelnde Nächstenliebe und Hilfsbereitschaft lassen sich unschwer als Reportage thematisieren. Gleiches gilt für Ehekonflikte und Versagen an gefährdeten Mitmenschen, für unterlassene Hilfe an Gastarbeitern, Kranken, psychisch belasteten Mitmenschen, für egoistische Richtungsstreitigkeiten in der Kirche selbst, für vorschnelle Verurteilungen Andersdenkender ebenso wie für das Schuldigwerden ohne eigene Schuldabsicht, jener Schuld, an der wohl am schwersten zu tragen ist, weil Sühne im rechtlichen Sinn nicht möglich ist.

Dies alles muß in der Kirchenzeitung seinen Platz haben, weil es in den weltlichen Kommunikationsmitteln höchstens als Sensation vermarktet wird - ohne dem Leser von Nutzen zu sein, weil er sich nicht angesprochen fühlt und weil Vergebung in der Regenbogenpresse nur zur Schnulze gedeiht. Die Sünde aber ist kein Unterhaltungsthema, wohl aber in der Kirchenzeitung eine Reportage wert. 


\section{Probieren gebt über Studieren}

Daß das Probieren über das Studieren geht, ist ein fester Bestandteil der Volksweisheit, und so hat der Verfasser im Januar 1978 bei einem Seminar des katholischen Instituts zur Förderung publizistischen Nachwuchses für Volontäre von Kirchenzeitungen in Augsburg die Probe auf das dargelegte Exempel gemacht. In knapp zwei Tagen sollte jeder der 15 Volontäre (darunter erfreulich viele Damen, was für die Zukunft der Kirchenpresse hoffen läßt) eine Reportage über einen Aspekt aus dem Gesamtthema „Pastorale Aufgaben in Augsburg heute" schreiben.

Das Engagement der Volontäre verdient besondere Anerkennung, das erzielte Ergebnis allerdings nicht weniger. Man zog aus, um Meßner, Pfarrhaushälterinnen, Betriebsseelsorger, Gemeindeassistenten, Laienmissionare in einer Reportage darzustellen. Die Okumene, ein katholisches Landschulheim, ein ökumenisches Lebenszentrum wurden ebenfalls als Thema gewählt. Und die Aufgabe, die vom Verfasser als besonders schwierig angesehen wurde, brachte ihm nach kurzer Zeit nur eine "freundliche Verachtung ${ }^{c}$ ein: Eine Volontärin sollte eine Reportage darüber schreiben, welche Schwierigkeiten sich ergeben, wenn man als einfacher Gläubiger einfach mal mit dem Bischof sprechen will. Die junge Kollegin saß nur wenig später im Ordinariat der Diözese Augsburg Weihbischof Rudolf Schmid gegenïber, der sie als "Jurastudentin " empfangen hatte und sie dann belehrte, daß die Bischöfe allen Vorurteilen zum Trotz - sie wurden später in einer Straßenbefragung „Würden Sie gern mal mit ihrem Bischof persönlich sprechen?“ bestätigt - die Nähe und das Gespräch mit den Gläubigen suchen.

Die meisten Reportagen zeigten, daß solche Themen in dieser Stilform geradezu ideal für eine Kirchenzeitung sind und Kirche heute auch dem Fernstehenden nahebringen können, ja sein Interesse wecken.

\section{Resignation muß nicht sein}

In zahlreichen Redaktionen von Kirchenzeitungen hat sich Resignation ausgebreitet. Die Auflagen der 22 deutschen Bistumszeitungen haben im letzten Jahrzehnt schwere Einbrüche hinnehmen müssen, und ob sich die gegenwärtige Gesamtauflage von 1,9 Millionen Exemplaren halten läßt, ist fraglich. Denn die treuesten Leser sind die älteren und alten Kirchenbesucher - sie werden zwangsläufig immer älter und damit sinkt die Auflage der Kirchenzeitungen. Die Verantwortlichen sorgen sich verständlicherweise um den halbwegs wirtschaftlichen Weg in die Zukunft. Sie suchen nach neuen Modellen, die notwendig sind.

Doch - davon ist der Verfasser überzeugt - die Möglichkeiten journalistischer Arbeit unter den gegebenen Bedingungen der Kirchenzeitungsredaktionen sind längst nicht ausgeschöpft. Eine Möglichkeit, wohlbemerkt eine, ist die Erinnerung an die Chancen, die in den journalistischen Stilformen selbst liegen. Anhand des Beispiels von Bericht und Reportage sollte darauf nou aufmerksam gemacht werden, um der Resignation $\mathrm{zu}$ wehren - bei Journalisten und Lesern von Kirchenzeitungen gleichermaßen. Denn die Kirchenzeitung, die katholische wie die evangelische, hat sich nicht selbst überlebt. Sie ist notwendig für die Kirche und die Welt, für communio et progressio - vielleicht bald notwendiger denn je. 
The place and role of reports and features in a Church paper is discussed. The expectation of Communio et Pregressio that the Catholic Press should be "a glass that reflects the world and a light to show it the way" (138) are largely unfulfilled. Journalistic forms and styles are not aims in themselves. Moreover, Church papers have to use different journalisticforms to report on the variety in the Church. Church communications should not be limited to pulpit language nor to Church jargon. Full use of all journalistic forms of expression has to be made, and differences and opposing positions should not be excluded. Despite decreasing circulation, thre is no reason to be pessimistic. The possibilities for all kinds of journalistic work are afforded within the Church Press and are certainly not fully used yet. The Church paper is not outdated: it is a need for the Church and the world.

\section{R É S U M É}

L'auteur cherche à ordonner lieu et fonction de l'exposé et du reportage dans le journal ecolésiastique. Il prétend que l'ordre de "Communio et Progressio" à la presse catholique: d'être "le miroir qui reflète le monde et la lumière qui lui montre le chemin" n'est encore qu'un but lointain. Il défend la thèse que les formes de style journalistiques ne sont pas une fin en soi et que le journal ecclésiastique lui-même a le devoir d'informer de la diversité de l'Eglise avec la diversité des formes de style journalistipues. Il se rapporte en cela à des garants ecclésiastiques faisant autorité et admoneste l'homélie de même que le jargon, et parle net une langue journalistique claire qui n'épargne pas non plus les conflits et les différentes positions. Malgré la diminution du nombre des lecteurs, assure l'auteur, la résignation est déplacé. Il tient les possibilités d'un travail journalistique, même aux conditions de rédactions de journaux ecclésiastiques, pour loin d'être épuisées. Le journal ecclésiastique n'est pas périmé. Il est nécessaire à l'Eglise et au monde.

\section{R E S UMEN}

El autor intenta encontrar lugar y sentido al relato y al reportaje en la prensa diocesana. Afirma que sigue siendo una meta lejana el encargo dado por "Communio et Progressio“ a la prensa católica: "ser espejo que refleje el mundo y luz que le muestre el camino". Defiende la tesis de que las formas estilísticas periodísticas no son sólo fin absoluto y que también la prensa diocesana tiene el cometida de informar sobre las diversas caras de la Iglesia con diversos estilos periodísticos. Cita en este contexto representativas autoridades eclesiásticas, destierra el lenguaje del púlpito tanto como la jerga y se pronuncia por el lenguaje periodístico, que tampoco ahorra conflictos ni posturas contrapuestas. A pesar del receso del número de lectores, el autor enfatiza que no hay motivo para la resignación. Considera que, incluso en las circunstancias características de las redacciones de la prensa diocesana, aún no se agotaron las posibilidades del trabajo periodístico. La prensa diocesana no es algo anticuado. Es necesaria para la Iglesia y el mundo. 